\title{
Checking Strong Optimality of Interval Linear Programming
}

\author{
Jia-Jia Luo \\ School of Science, Hangzhou Dian zi University \\ Hangzhou, China \\ E-MAIL: 420155682@qq.com
}

\begin{abstract}
In this paper, we will discusse the strong optimality of general interval linear program (IvLP), that is, the IvLP has an optimal solution for each realization, and some necessary and sufficient conditions are established, based on the duality theory.
\end{abstract}

\section{Keywords- optimal solution; strongly feasible; duality;}

\section{INTRODUCTION}

Many practical problems are solved by linear program (LP). Since real-life proble ms are subject to uncertainties due to errors, measurements and estimations, we have to reflect it in linear programming methodology and decision making. Interval computations is a popular way for treating uncertainties in data measurements. The optimality of given vectors has be learned by many researchers, see, e.g. [2],[3],[4],[6],[9]. In this paper, we will discuss the strong optimality of IvLP, that is, each realization of the IvLP has an optimal solution, and propose a necessary and sufficient condition, based on the duality theory.

Throughout the paper, we follow the definitions and notations gives in [6], which provides a detailed survey on solvability of system of interval linear equations and inequalities.

Let $\underline{A}, \bar{A} \in R^{m \times n}$, and $\underline{A} \leq \bar{A}$. The set of matrices

$$
\mathbf{A}=[\underline{A}, \bar{A}]=\{\underline{A} \leq A \leq \bar{A}\}
$$

is called an interval matrix, where matrices $\underline{A}, \bar{A}$ are referred to as its lower and upper bounds. Similarly, we define an interval vector $\mathbf{b}$ as an one column interval matrix where $\underline{b}, \bar{b} \in R^{m}$.

$$
\mathbf{b}=[\underline{b}, \bar{b}]=\{\underline{b} \leq b \leq \bar{b}\},
$$

Denote by $A_{c}$ and $A_{\Delta}$ the center and radius matrices given by

$$
A_{c}=\frac{1}{2}(\underline{A}+\bar{A}), A_{\Delta}=\frac{1}{2}(\bar{A}-\underline{A})
$$

respectively. Then $\mathbf{A}=\left[A_{c}-A_{\Delta}, A_{c}+A_{\Delta}\right]$. Similarly, the center and radius vectors are defined as

$$
b_{c}=\frac{1}{2}(\underline{b}+\bar{b}), b_{\Delta}=\frac{1}{2}(\bar{b}-\underline{b})
$$

respectively. Then $\mathbf{b}=\left[b_{c}-b_{\Delta}, b_{c}+b_{\Delta}\right]$.

Let $Y_{m}$ be the set of all $\{-1,1\}$ m-dimensional vectors, i.e.

$$
Y_{m}=\left\{y \in R^{m}|| y \mid=e\right\},
$$

\section{Wei Li}

School of Science, Hangzhou Dian zi University, Hangzhou, China E-MAIL:weili@hdu.edu.cn

where $e=(1, \cdots, 1)^{T}$ is the m-dimensional vector of all 1 ' $\mathrm{s}$

For a given $y \in Y_{m}$, let

$$
T_{y}=\operatorname{diag}\left(y_{1}, \cdots, y_{m}\right)
$$

denote the corresponding diagonal matrix.

For a given interval matrix $\mathbf{A}=\left[A_{c}-A_{\Delta}, A_{c}+A_{\Delta}\right]$ and vectors $y \in Y_{m}$ and $z \in Y_{n}$, we introduce the following matrices:

$$
A_{y z}=A_{c}-T_{y} A_{\Delta} T_{z} .
$$

Similarly, for an interval vector $\mathbf{b}=\left[b_{c}-b_{\Delta}, b_{c}+b_{\Delta}\right]$ and vector $y \in Y_{m}$, we define vectors

$$
b_{y}=b_{c}+T_{y} b_{\Delta}
$$

\section{PRELIMINARIES}

\section{A. Linear Program: Duality}

Consider the LP problem

$$
\min c^{T} x \text { subject to } x \in M(A, b) \text {, }
$$

where $M(A, b)$ is the feasible set characterized by a linear system. The LP (1) is called a primal LP problem, and we can formulate its dual linear programming (DLP) problem as

$$
\max b^{T} y \text { subject to } y \in M(A, c) \text {. }
$$

For example,

(1) the DLP problem of the LP

$$
\min c^{T} x \text { subject to } A x=b, x \geq 0
$$

is

(2) the DLP problem of the LP

$$
\min c^{T} x \text { subject to } A x \leq b
$$

is

$$
\max b^{T} y \text { subject to } A^{T} y=c, y \leq 0 \text {; }
$$

(3) the DLP problem of the LP

$$
\min c^{T} x \text { subject to } A x \leq b, x \geq 0
$$


$\max b^{T} y$ subject to $A^{T} y \leq c, y \leq 0$.

For a given LP (1) we introduce two the values

$$
\begin{aligned}
& f(A, b, c)=\inf \left\{c^{T} x \mid x \in M(A, b)\right\}, \\
& g(A, b, c)=\sup \left\{b^{T} y \mid y \in M(A, c)\right\},
\end{aligned}
$$

and we call them the optimal value of (1) and the optimal value of (2). Exactly one of the following three cases may occur.

a) if $f(A, b, c)$ is finite, then there exist a feasible solution $x^{*}$ of (1) satisfying $f(A, b, c)=c^{T} x^{*}$. Such an $x^{*}$ is called an optimal solution of (1).

$b)$ if $f(A, b, c)=-\infty$, we call (1) unbounded.

$c$ ) if there is no $f(A, b, c)$, then the set of feasible solutions of (1) is empty.

Theorem 2.1 [6] If $f(A, b, c)<\infty$ or $g(A, b, c)>-\infty$, then

$$
f(A, b, c)=g(A, b, c) .
$$

The formal equality covers two qualitative issues:

d) If one of the problems (1) and (2) has an optimal solution, then so does the second one and the optimal values of both problems are equal.

e) If one of the problems (1) and (2) is unbounded, the second one is infeasible.

In this paper, "the problem is feasible" means the feasible set $M(A, b)$ is not empty, and "infeasible" in the opposite case.

\section{B. Interval Linear Program}

Let $\mathbf{A} \in I R^{m \times n}, \mathbf{b} \in I R^{m}$ and $\mathbf{c} \in I R^{n}$ be given. By an IvLP problem we mean a family of LP (1), where $A \in \mathbf{A}, b \in \mathbf{b}, c \in \mathbf{c}$. We write it in short as

$$
\operatorname{minc}^{T} x \text { subject to } x \in M(\mathbf{A}, \mathbf{b}) \text {. }
$$

Let us focus on the feasible set description for a while. In the IvLP theory, one of the following canonical forms

(A) $M(\mathbf{A}, \mathbf{b})=\left\{x \in R^{n} ; \mathbf{A} x=\mathbf{b}, x \geq 0\right\}$,

(B) $M(\mathbf{A}, \mathbf{b})=\left\{x \in R^{n} ; \mathbf{A} x \leq \mathbf{b}\right\}$,

(C) $M(\mathbf{A}, \mathbf{b})=\left\{x \in R^{n} ; \mathbf{A} x \leq \mathbf{b}, x \geq 0\right\}$

is usually assumed.

Theorem 2.2 [6] A system $\mathbf{A} x=\mathbf{b}$ is strongly feasible if and only if for each $z \in Y_{m}$ the system

has a nonnegative solution.

$$
A_{z e} x=b_{z}
$$

Theorem 2.3 [6] A system $\mathbf{A} x \leq \mathbf{b}$ is strongly solvable if and only if the system

is solvable.

$$
\bar{A} x^{1}-\underline{A} x^{2} \leq \underline{b}, x^{1}, x^{2} \geq 0
$$

In this paper, " $\mathbf{A} x=\mathbf{b}$ is strongly feasible" means each realization of $\mathbf{A} x=\mathbf{b}$ has nonnegative solutions, and "A system $\mathbf{A} x \leq \mathbf{b}$ is strongly solvable" means each realization of $\mathbf{A} x \leq \mathbf{b}$ has solutions,

In next section, we will discuss the IvLP with three kinds of constraint conditions above, and propose some necessary and sufficient conditions to check the IvLP has an optimal solution for each realization.

\section{MAIN RESUltS}

Theorem 3.1 Each realization of the Iv LP

$$
\operatorname{minc}^{T} x \text { subject to } \mathbf{A} x=\mathbf{b}, x \geq 0
$$

has an optimal solution if and only if the system

$$
\bar{A}^{T} y^{1}-\underline{A}^{T} y^{2} \leq \underline{c}, y^{1}, y^{2} \geq 0
$$

is solvable, and for each $z \in Y_{m}$ the system

$$
A_{z e} x=b_{z}
$$

has a nonnegative solution.

Proof "If": Let the system(6) has a nonnegative solution for each $z \in Y_{m}$. From Theorem 2,2 we have that $\mathbf{A} x=\mathbf{b}$ is strongly feasible, that is, each realization of $\mathbf{A} x=\mathbf{b}$ has nonnegative solutions. Then for each $A \in \mathbf{A}, b \in \mathbf{b}, c \in \mathbf{c}$, the LP problem

$$
\min c^{T} x \text { subject to } A x=b, x \geq 0
$$

is feasible. From the analysis above, the LP (7) has an optimal value $f(A, b, c)$ which may be infinite. Now assume $f(A, b, c)$ is infinite, then we have the DLP problem

$$
\max b^{T} y \text { subject to } A^{T} y \leq c
$$

of (7) is infeasible. But the system (5) is solvable, from Theorem 2.3 we now that $\mathbf{A}^{T} y \leq \mathbf{c}$ is strong solvable, that is, for each $A \in \mathbf{A}, c \in \mathbf{c}, A^{T} y \leq c$ has solutions. Thus, the DLP (8) is feasible. This is a contradiction. So the optimal value $f(A, b, c)$ of (7) is finite, which means it has an optimal solution. Hence each realization of (4) has an optimal solution.

"Only if": Let each realization of (4) has an optimal solution (or optimal value), then it is feasible. Hence the system $\mathbf{A} x=\mathbf{b}, x \geq 0$ is strongly solvable, that is, $\mathbf{A} x=\mathbf{b}$ is strongly feasible. From Theorem 2.2 we have the system(6) has a nonnegative solution for each $z \in Y_{m}$. At the same time, from Theorem 2.1 the DLP problem of each realization of (4) has an optimal value, which means it is feasible, hence the dual Iv LP problem

$$
\max \mathbf{b}^{T} y \text { subject to } \mathbf{A}^{T} y \leq \mathbf{c}
$$


of (4) is strongly feasible which means the system $\mathbf{A}^{T} y \leq \mathbf{c}$ is strong solvable, and from Theorem 2.3 the system (5) is solvable

This completes the proof of the theorem.

Theorem 3.2 Each realization of the

$$
\operatorname{minc}^{T} x \text { subject to } \mathbf{A} x \leq \mathbf{b}
$$

has an optimal solution if and only if the system

$$
\bar{A} x^{1}-\underline{A} x^{2} \leq \underline{b}, x^{1}, x^{2} \geq 0
$$

is solvable, and for each $z \in Y_{n}$ the system

$$
\left(A^{T}\right)_{z e} x=-c_{z}
$$

has a nonnegative solution.

Proof "If": Let the system (10) is solvable. From Theorem 2.3 we know $\mathbf{A} x \leq \mathbf{b}$ is strongly solvable, that is, the IvLP (9) is strongly feasible. Then for each $A \in \mathbf{A}, b \in \mathbf{b}, c \in \mathbf{c}$ the LP problem

$$
\min c^{T} x \text { subject to } A x \leq b
$$

is feasible. From the analysis above, the LP (12) has an optimal value $f(A, b, c)$ which may be infinite. Now assume $f(A, b, c)$ is infinite, then we have the DLP problem

$$
\max b^{T} y \text { subject to } A^{T} y=c, y \leq 0
$$

of (12) is infeasible. But for each $z \in Y_{n}$ the system(11) has a nonnegative solution, then from Theorem 2.2 we have $\mathbf{A}^{T} x=-\mathbf{c}$ is strong feasible. Let $x=-y$, then we have $\mathbf{A}^{T} y=\mathbf{c}, y \leq 0$ is strongly solvable, that is, the DLP (13) is feasible. This is a contradiction. Thus, the optimal value $f(A, b, c)$ of (12) is finite, which means it has an optimal solution. Hence each realization of (9) has an optimal solution.

"Only if": Let each realization of (9) has an optimal solution (or optimal value), then it is feasible. Hence the system $\mathbf{A} x \leq \mathbf{b}$ is strongly solvable. From Theorem 2.3 we have the system (10) is solvable. At the same time, from Theorem 2.1 the DLP problem of each realization of (9) has an optimal value, which means it is feasible, hence the dual IvLP problem

$$
\max \mathbf{b}^{T} y \text { subject to } \mathbf{A}^{T} y=\mathbf{c}, y \leq 0
$$

of (9) is strongly feasible. That is, the system $\mathbf{A}^{T} y=\mathbf{c}, y \leq 0$ is strong solvable. Let $y=-x$, then we have the system $\mathbf{A}^{T} x=-\mathbf{c}, x \geq 0$ is strong solvable, that is, the system $\mathbf{A}^{T} x=-\mathbf{c}$ is strongly feasible, and from Theorem
2.2 the system (11) has a nonnegative solution for each $z \in Y_{n}$.

This completes the proof of the theorem.

Theorem 3.3 Each realization of the

$$
\operatorname{minc}^{T} x \text { subject to } \mathbf{A} x \leq \mathbf{b}, x \geq 0
$$

has an optimal if and only if the systems

$$
\bar{A} x \leq \underline{b}, x \geq 0
$$

$$
\underline{A}^{T} y \leq \underline{c}, y \leq 0
$$

have solutions $\bar{x}, \bar{y}$.

Proof "If": Let the two systems have solutions $\bar{x}, \bar{y}$. For each $A \in \mathbf{A}, b \in \mathbf{b}, c \in \mathbf{c}$, the LP problem

$$
\min c^{T} x \text { subject to } A x \leq b, x \geq 0
$$

is a realization of (14). We show that the LP (17) has an optimal solution. Because the system (15) has a solution $\bar{x}$, we know that $A \bar{x} \leq \bar{A} \bar{x} \leq \underline{b} \leq b, \bar{x} \geq 0$ holds. Hence the LP (17) is feasible. From the analysis above, the LP (17) has an optimal value $f(A, b, c)$ which may be infinite. Now assume $f(A, b, c)$ is infinite, then we have the DLP problem

$$
\max b^{T} y \text { subject to } A^{T} y \leq c, y \leq 0
$$

of (17) is infeasible. But the system (16) has a solution $\bar{y}$, then we know that $A^{T} \bar{y} \leq \underline{A}^{T} \bar{y} \leq \underline{c} \leq c, \bar{y} \leq 0$ holds. Hence the DLP (18) is feasible. This is a contradiction. Thus, the optimal value $f(A, b, c)$ of (17) is finite, which means it has an optimal solution. Hence each realization of (14) has an optimal solution.

"Only if": Let each realization of (14) has an optimal solution, then it is feasible. Thus, the LP problems

$$
\begin{aligned}
& \min \bar{c}^{T} x \text { subject to } \bar{A} x \leq \underline{b}, x \geq 0 \\
& \min \underline{c}^{T} x \text { subject to } \underline{A} x \leq \underline{b}, x \geq 0
\end{aligned}
$$

are feasible, which means the system (15) has solution $\bar{x}$. The DLP of (19) is

$$
\max \underline{b}^{T} y \text { subject to } \underline{A}^{T} y \leq \underline{c}, y \leq 0 .
$$

Because the LP (19) has an optimal solution, that is, it has a finite optimal value, from Theorem 2.1 we know that the 
DLP (20) has an finite optimal value, hence it is feasible, that is, the system (16) has solution $\bar{y}$.

This completes the proof of the theorem.

\section{ILLUSTRATIVE EXAMPLES}

Now we make an example to explain Theorem 3.3.

Example 1 Consider the IvLP problem

$$
\begin{aligned}
\min & {[-2,-1] x_{1}+[-3,-1] x_{2}+[-3,0] x_{3} } \\
\text { s.t } & {[1,2] x_{1}+[0,1] x_{2}+[2,3] x_{3} \leq[1,3], } \\
& 2 x_{1}+x_{2}+[-2,0] x_{4} \leq[0,1], \\
& x_{1}, x_{2}, x_{3} \geq 0 .
\end{aligned}
$$

Now construct the corresponding systems (15) and (16). Note that

$$
\bar{A}=\left(\begin{array}{lll}
2 & 1 & 3 \\
2 & 1 & 0
\end{array}\right), \underline{A}=\left(\begin{array}{ccc}
1 & 0 & 2 \\
2 & 1 & -2
\end{array}\right), \underline{b}=\left(\begin{array}{l}
1 \\
0
\end{array}\right), \bar{c}=\left(\begin{array}{l}
-2 \\
-3 \\
-3
\end{array}\right) .
$$

Thus, the linear system (15) is

$$
\begin{aligned}
& 2 x_{1}+x_{2}+3 x_{3} \leq 1, \\
& 2 x_{1}+x_{2} \leq 0, \\
& x_{1}, x_{2} \geq 0 .
\end{aligned}
$$

It is obvious that $x=(0,0,0)^{T}$ is a solution to the linear system above.

The linear system (16) is

$$
\begin{aligned}
& y_{1}+2 y_{2} \leq-2, \\
& y_{2} \leq-3, \\
& 2 y_{1}-2 y_{2} \leq-3, \\
& y_{1}, y_{2} \leq 0 .
\end{aligned}
$$

It is obvious that $y=(-5,-3)^{T}$ is a solution to the linear system above.

From Theorem 3.3, the IvLP (21) has an optimal solution for each realization. In this problem, we can not take all real matrices and real vectors to check it, so we take two combinations of statistics arbitrarily.

(1) take

$$
A=\left(\begin{array}{lll}
1 & 1 & 2 \\
2 & 1 & 0
\end{array}\right), \quad b=\left(\begin{array}{l}
1 \\
0
\end{array}\right), c=\left(\begin{array}{c}
-1 \\
-1 \\
0
\end{array}\right),
$$

then we have the LP problem

$$
\begin{array}{ll}
\min -x_{1}-x_{2} & \\
\text { s.t } & x_{1}+x_{2}+2 x_{3} \leq 1, \\
& 2 x_{1}+x_{2} \leq 0, \\
& x_{1}, x_{2}, x_{3} \geq 0 .
\end{array}
$$

By the Lingo, the LP above have an optimal solution $\bar{x}=(0,1,0)^{T}$.
(2) take

$$
A=\left(\begin{array}{lll}
1 & 0 & 3 \\
2 & 1 & 0
\end{array}\right), \quad b=\left(\begin{array}{l}
3 \\
1
\end{array}\right), c=\left(\begin{array}{l}
-1 \\
-1 \\
-1
\end{array}\right),
$$

then we have the LP

$$
\begin{array}{cc}
\min -x_{1}-x_{2}-x_{3} \\
\text { s.t } \quad x_{1}+3 x_{3} \leq 3, \\
2 x_{1}+x_{2} \leq 1, \\
x_{1}, x_{2}, x_{3} \geq 0 .
\end{array}
$$

By the Lingo, the LP above have an optimal solution $\bar{x}=(0,1,1)^{T}$.

\section{CONCLUSIONS}

This paper discusses the optimality of the IvLP problems with three kinds of constraint conditions, that is, the IvLP has an optimal solution for each realization and proposes some necessary and sufficient conditions to check it based on the duality theory. The systems (15) and (16) are polynomially solvable since each of them suffices to test feasibility of a real-valued linear system. Nevertheless, the "bad boy" is the system of equations as we have to check feasibility of $2^{m}$ systems in Theorem 3.1 and of $2^{n}$ systems in Theorem 3.2. Indeed, it was shown [6] that checking this property is NPhard. That how to propose a better necessary and sufficient condition is open problem. This should be a subject of further research.

\section{ACKNOWLEDGMENT}

This project was supported by the National Natural Science Foundation of China (Grant No. 61003194, 11171316) and Zhejiang province university students' innovative and undertaking incubator program (R407061).

\section{REFERENCES}

[1] M. Inuiguchi, H. Ichihashi and Y Kume, "Relationship between modality constrained programming problems and various fuzzy mathematical problems", Fuzzy Sets and Systems, Vol. 49, No. 3, pp. 243-259, Aug. 1992.

[2] M. K. Luhandjula, "Fuzzy optimization: an appraisal", Fuzzy Sets and Systems, Vol. 30, No. 3, pp. 257-282, May 1989.

[3] M. Sakawa, Fuzzy Sets and Interactive Multiobjective Optimization, Plenum Press, New York, 1993.

[4] Wei Li, Xiaoli Tian, "Numerical solution method for general interval quadratic programming", Applied Mathematics and Computation, Vol. 202, No. 2, pp. 589-595, Aug. 2008.

[5] Wei Li, Xiaoli Tian, "Fault detection in discrete dynamic systems with uncertainty based on interval optimization", Mathematical modelling and analysis, Vol. 16, No. 4, pp. 549-557, sep. 2011.

[6] M. Fiedler, J. Nedoma, J. Ramik, J. Rohn, K. Zimmermann, Linear optimization problems within exact data, Springer, New York, 2006.

[7] J. Rohn, "Strong solvability of interval linear programming problems", Computing, Vol. 26, No. 1, pp. 79-82, Mar. 1981.

[8] J. Rohn, "Systems of linear interval equations", Linear Algebra Applications, Vol. 126, pp. 39-78, Dec. 1989.

[9] M. Hlad'ik, Interval Linear Programming: A Survey, In: Linear Programming-New Frontiers in Theory and Applications, Nova Science Publishers, New York, pp. 85-120, 2011. 